\title{
Aneurismas renales múltiples que involucran rama principal. Resolución percutánea con técnica combinada de microcoils y stent diversor de flujo
}

\author{
Multiple renal aneurysms involving main branch. Percutaneous resolution \\ with combined technique of microcoils and flow diversion stent
}

Juan Mieres, R. Farfán, H. Pavlovsky, Y. Navarro, S. Burda

\begin{abstract}
RESUMEN
Se ha observado que los aneurismas de la arteria renal (AAR) son raros aunque potencialmente fatales. En los aneurismas hay una debilidad de toda la pared, mientras que en los pseudoaneurismas existe una alteración focal en una capa que causa la evaginación de un saco. Si bien existe cierta controversia sobre el tiempo de resolución de AAR con respecto a su tamaño en pacientes asintomáticos, existe un cierto consenso de que cuando superan los $2 \mathrm{~cm}$, deben excluirse. Presentamos un paciente con dolor lumbar derecho durante meses de evolución que se somete a una tomografía computarizada que muestra múltiples AAR, uno de ellos de tamaño 26x12 mm proximal y otro de tamaño $8 \times 6 \mathrm{~mm}$ distal, en este primero se observa la salida de rama inferior de tamaño signficativo. Se realiza intervención renal selectiva, y con microcatéter se colocan cinco microcoils, en el aneurisma proximal, y dos microcoils en el aneurisma distal. Posteriormente, se implantó un stent diversor de flujo para cubrir el techo de estos dos aneurismas hasta el tercio distal del tronco de la arteria renal derecha. Seis meses después se le realizó una angiografía de seguimiento, confirmando la exclusión completa con preservación de la rama renal inferior. Combinado con microcoils y el stent derivador de flujo es una técnica que combina el tratamiento del AAR para preservar las ramas principales. Este stent multicapa genera diferentes flujos en el aneurisma y en las ramas principales, que genera una trombosis del aneurisma con preservación de sus ramas principales.
\end{abstract}

Palabras claves: aneurisma renal (AAR), stent diversor de flujo, coils, tratamiento percutáneo.

\begin{abstract}
Renal Artery (RAA)) aneurysms have been observed to be rare, although potentiaIly fatal, in the aneurysms there is a weakness of the entire wall while in the pseudoaneurysms there is a focal disruption in a layer causing the evagination of a sack.Although there is some controversy about the resolution time of RAA with respect to its size in asymptomatic patients, there is a certain consensus that when they exceed 2 $\mathrm{cm}$, they should be excluded.Patient with right lumbar pain for months of evolution undergoes a CT scan that show multiple RAA, one of them of the size $26 \times 12 \mathrm{~mm}$ proximal and a second of the size $8 \times 6 \mathrm{~mm}$ distal, this first involves the exit of the significant lower renal branch. Selective renal intervention is performed, and with microcatheter and five microcoils, in the proximal aneurysm and two microcoils in distal aneurysm are placed. Subsequently, a Flow- diverting stent was implanted to cover the roof of these two aneurysms up to the distal third of the trunk of the Right Renal Artery. Six months later, angiography was performed, confirming complete exclusion with preservation the renal branch.Combined with coils and Flow- diverting stent is a technique that combines the treatment of the RAA to preserve main branches. This multilayer stent generates different flows in the aneurysm and in the main branches. What generates a thrombosis of the aneurysm with preservation of its main branches.
\end{abstract}

Key words: renal artery aneurysm (RAA), flow diversion stent, coils, percutaneous treatment.

\section{INTRODUCCIÓN}

Se han observado que los aneurismas de la arteria renal (AAR) son poco frecuentes aunque potencialmente fatales, como muestra la primera publicación de Rouppe ${ }^{1}$ en 1770 , quien describió la muerte de un pescador que cayó sobre su flanco derecho y la autopsia reveló la rotura de un pseudoaneurisma renal. Aunque existe cierta controversia sobre el tiempo de resolución de los AAR con respecto a su tamaño en pacientes asintomáticos, hay cierto consenso que cuando superen los 2 $\mathrm{cm}$ hay que excluirlos ${ }^{2}$. En los aneurismas existen una debilidad de toda la pared mientras en los pseudoaneurismas hay una disrupción focal en una capa que causa la evaginación de un saco. ${ }^{3}$ En el trauma cerrado, con desplazamiento anterior en riñones móviles, esta rápida desaceleración causa fractura de la íntima, predisponiendo a la disección subintimal ${ }^{4}$, que produce una degeneración hacia aneurisma.

\section{OBJETIVOS}

Presentar la resolución por vía percutánea con técnica com-

1. Centro CECI. Buenos Aires y San Isidro, Rep. Argentina

$\checkmark$ Correspondencia: Dr. Juan Mieres. Centro CECI (Sanatorio Otamendi y Sanatorio Las Lomas).Buenos Aires y San Isidro,Argentina.jmieres@centroceci.com.ar Los autores declaran no tener conflictos de intereses

Recibido: 15/02/2019|Aceptado: 18/03/2019 binada de coils y stent diversor de flujo para preservar la rama principal que emerge de AAR. Descripción de la técnica y su seguimiento angiográfico a seis meses del procedimiento.

\section{CASO CLÍNICO}

Paciente de 50 años, con sobrepeso, con dolor lumbar derecho de meses de evolución, por lo que se realiza ecografía abdominal que sugiere imagen compatible con AAR, posteriormente se somete a Tomografía axial computarizada de múltiples cortes y resonancia magnética nuclear que comprueban que presenta AAR múltiples, uno de 26x12 mm y uno segundo de $8 \times 6 \mathrm{~mm}$, este primero involucra la salida de la rama renal inferior de gran tamaño (Figura 1). Con estos antecedentes concurre al departamento de Hemodinamia, donde se realiza una arteriografía renal selectiva, donde podemos comprobar estos hallazgos.

Después de este estudio tomográfico y angiográfico se discutió con en nuestro servicio las diferentes posibilidades de exclusión tanto por vía percutánea como por vía quirúrgica. Se decide tratamiento percutáneo y programa el procedimiento con una precarga de clopidogrel de $300 \mathrm{mg} 24$ horas al procedimiento. Se procede a la intervención renal selectiva, y con microcatéter tipo Neuroslider 17 (Acandis, Pforzheim, Alemania) y se implantan 5 microcolis tipo Barricade ${ }^{\text {Tx }}$ (Invine, California, EE.UU.) en aneurisma proximal y 2 microcoils tipo Barricade ${ }^{\text {rx }}$ (Invine, California, EE.UU.) en aneurisma distal. Posteriormente se implanta un stent diversor de flujo tipo Derivo (Acandis, 


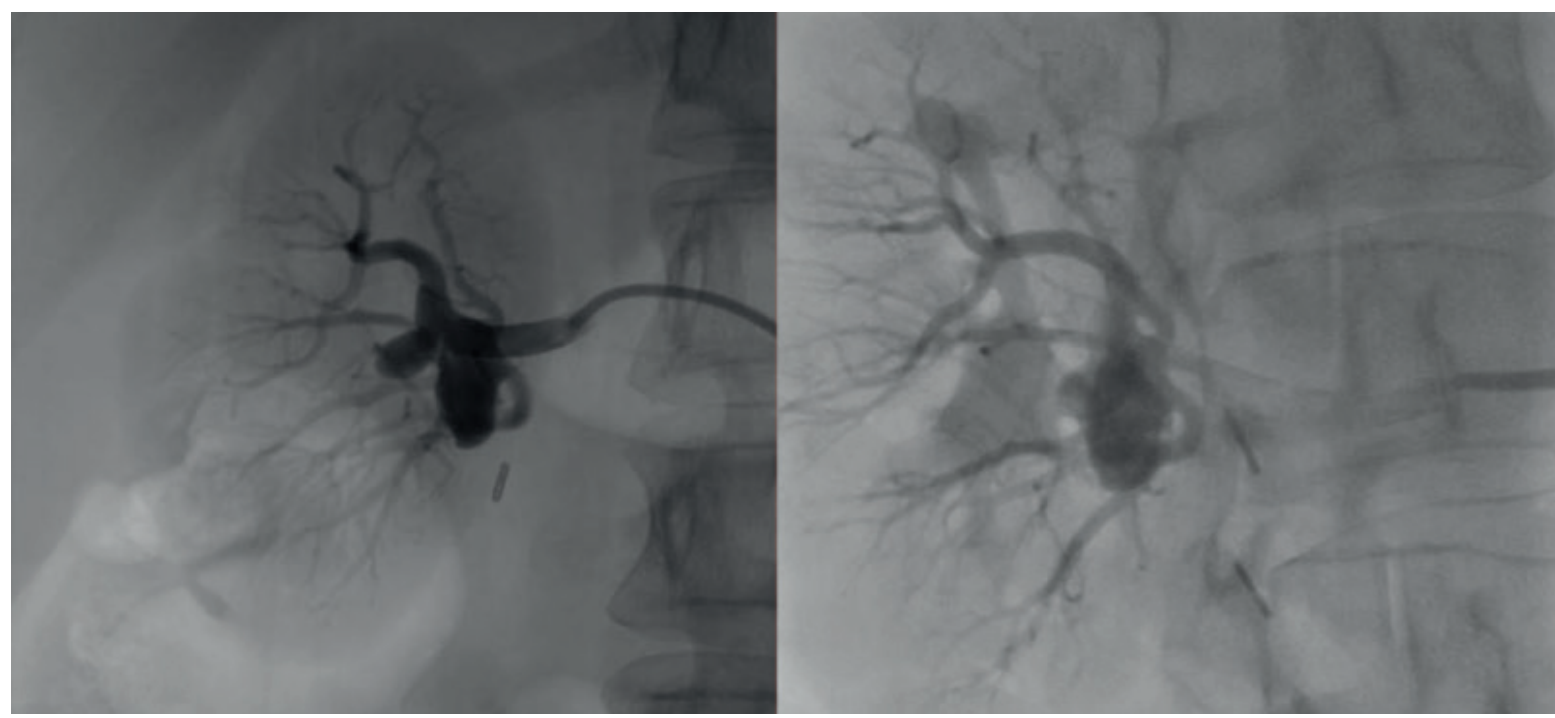

Figura 1. Se observan dos aneurismas renales, el mas proximal involucra la salida de una rama renal significativa.

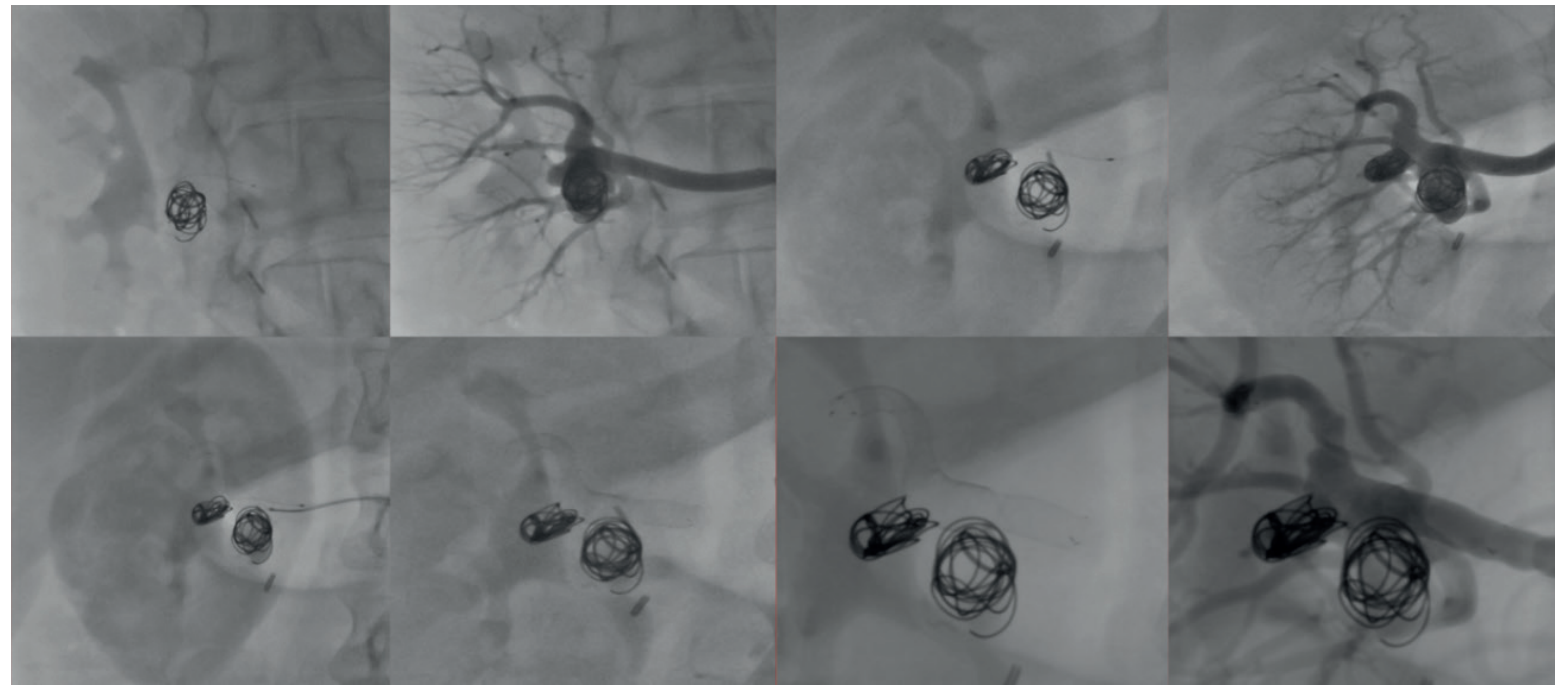

Figura 2. Tratamiento. Podemos observar la embolización del aneurisma proximal conmicrocoils, tipo Barricade ${ }^{\mathrm{TM}}$, y angiografía de control. Tratamiento del Segundo aneurisma con microcoilstipoBarricade ${ }^{\text {TM }}$.En la tercera etapa se coloca un StentDiversor de flujotipo, DERIVO ${ }^{\circledast}$, observándose luego la permeabilidad de la rama renal inferior.

Pforzheim, Alemania) de $6 \times 40 \mathrm{~mm}$, para cubrir el techo de estos dos aneurismas hasta el tercio distal del tronco de la arteria renal derecha (Figura 2). Fue dada de alta al día siguiente con clopidogrel $75 \mathrm{mg}$ /día, aspirina $100 \mathrm{mg} /$ día y atorvastatina $10 \mathrm{mg} /$ día. Se realiza control tomográfico a los dos meses de seguimiento donde se observa una exclusión casi completa con preservación de la rama renal inferior que estaba involucrado en el AAR medial. A los seis meses (Figura 3) se realiza angiografía de control constatándose exclusión completa del aneurisma medial y casi completa del distal, con preservación de la rama renal inferior que emerge de AAR medial. Se mantuvo el clopidogrel por 3 meses y la aspirina por al menos 6 meses.

\section{DISCUSIÓN}

Los datos epidemiológicos sugieren que los AAR ocurren aproximadamente en el $0,7 \%$ de la población cuando se lo analiza por los hallazgos en la tomografía 5 . La incidencia fue mayor en pacientes hipertensos $(2,5 \%)$, siendo especialmente elevada en pacientes con fibrodisplasias de la arteria renal $(10 \%)^{6}$. La edad media de presentación es entre los $40 \mathrm{y}$ 60 años, con predominio femenino. La localización más fre- cuente es la extraparenquimatosa, comprendiendo aproximadamente el $85 \%$ de todos los AAR. El $70 \%$ son saculares, el $20 \%$ fusiformes y el $10 \%$ con disección. Se presentan como patología bilateral en el $20 \%$ de los casos y aneurismas múltiples en $30 \%{ }^{7}$. Las etiologías más comunes son la displasia fibromuscular y la aterosclerosis, la mayoría de los cuales son extraparenquimatosos y afectan a la arteria renal principal (90\%). Los falsos aneurismas o pseudoaneurismas, en los que no se respetan todas las capas de la arteria, son usualmente de origen iatrogénico, traumático, infeccioso involucrando más frecuentemente las arterias parenquimatosas intrarrenales. La mayoría de las AAR son asintomáticos y se encuentran incidentalmente en estudios complementarios. ${ }^{8}$ El dolor abdominal, usualmente localizado en el flanco,puede estar presente, sobre todo en los pacientes con aneurismas causados por disección. La hematuria puede ser otra manifestación de la disección o ruptura del AAR. ' La obstrucción del sistema colector y el infarto renal son síntomas menos frecuentes del AAR. La ruptura del aneurisma es infrecuente y ocurre en menos del 3\% de los pacientes, manifestándose típicamente con signos y síntomas de abdomen agudo hasta franco $s h o c k^{10}$. La incidencia de ruptura es eleva- 


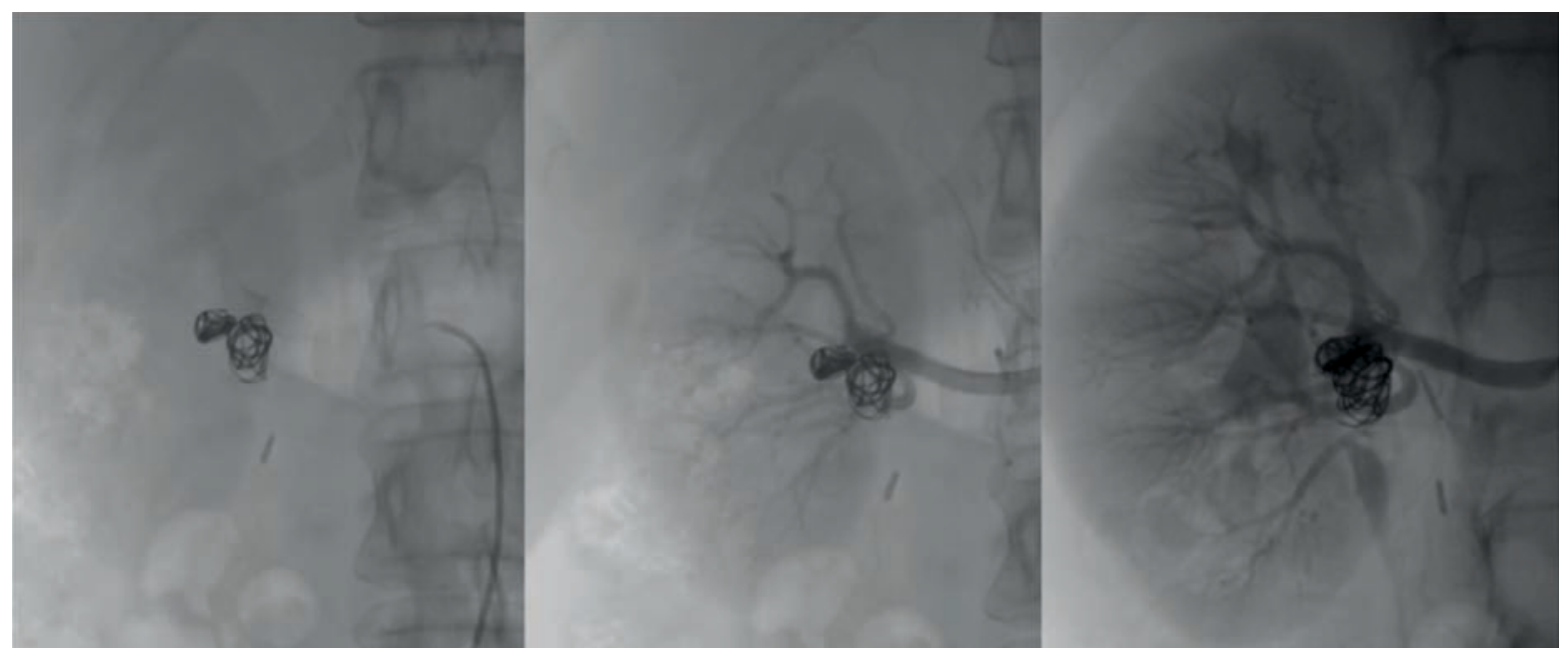

Figura 3. Seis meses de seguimiento. En el seguimiento se observa que la rama renal inferior se encuentra permeable, y que los aneurismas se encuentran excluidos.

da en aneurismas mayores de $2 \mathrm{~cm}$, particularmente en mujeres embarazadas con mortalidad materna alrededor del $50 \%$ y fetal del $80 \%$. Sin embargo, la mortalidad en pacientes no embarazadas es menor ocurriendo en $<10 \%$ de los ca$\operatorname{sos}^{11}$. En la actualidad, existe controversia en cuanto al tamaño en el que un AAR debe ser reparado en un paciente asintomático. Los expertos recomiendan reparación con diámetros que van desde 15 a $30 \mathrm{~mm}^{2}$, aunque en general se acepta que los aneurismas con un diámetro $>20 \mathrm{~mm}$ deben ser tratados. No obstante mujeres en edad fértil o embarazadas con evidencia de embolias y pacientes sintomáticos (con dolor, hipertensión resistente a tratamiento médico o hematuria) o aneurismas de rápida expansión deberían ser tratados con diámetros menores ${ }^{12}$. Los AAR asintomáticos,pequeños $\left(<2 \mathrm{~cm}\right.$ de diámetro) no suelen requerir tratamiento ${ }^{13}$.

Tradicionalmente, los AAR fueron tratados mediante cirugía abierta, ya sea por aneurismorrafia, reparación ex vivo, resección y derivación con Injerto, o con nefrectomía con una mor-

\section{BIBLIOGRAFÍA}

1. 1 Rouppe DL. Nova Acta Phys-Med Acad Nat Curios. 1770. iv:76.

2. 2Dzsinich C, Gloviczki P, McKusick MA, et al. Surgical management of renal artery aneurysm. Cardiovasc Surg. 1993 Jun. 1(3):243-7.

3. 3Calligaro KD, Dougherty MJ. Renovascular disease: aneurysms and arteriovenous fistulae. Cronenwett JL, Johnston KW, eds. Rutherford's Vascular Surgery. 8th ed. Philadelphia: Elsevier Saunders; 2014. Vol 2: 2326-34.

4. 4 Gewertz BL, Stanley JC, Fry WJ. Renal artery dissections. Arch Surg. 1977 Apr. 112(4):409-14.

5. 5 Zhang LJ, Yang GF, Qi J, Shen W. Renal artery aneurysm: diagnosis and surveillance with multidetector-row computed tomography. Acta Radiol. 2007 Apr. 48(3):274-9.

6. 6Stanley JC, Rhodes EL, Gewertz BL, et al. Renal artery aneurysms. Signifcance ofmacroaneurysms exclusive ofdissections and fibrodysplasticmural dilations. ArchSurg. 1975 Nov. 110(11):1327-33.

7. 7Bastounis E, Pikoulis E, Georgopoulos S, et al. Surgery for renal artery aneurysms: a combined series of two large centers. Eur Urol. 1998. 33(1):22-7.

8. 8 Henriksson C, Bjorkerud S, Nilson AE, et al. Natural history of renal artery aneurysm elucidated by repeated angiography and pathoanatomical studies. Eur Urol. 1985. 11(4):244-8.

9. 9HubertJPJr, Pairolero PC, Kazmier FJ. Solitary renal artery aneurysm. Surgery. 1980 Oct. 88(4):557-65.

10. 10 Cohen JR, Shamash FS. Ruptured renal artery aneurysms during pregnancy. J Vasc Surg. 1987 Jul. 6(1):51-9.

11. 11 Lumsden $A B$, Salam TA, Walton KG. Renal artery aneurysm: a report of 28 cases. CardiovascSurg. 1996 Apr. 4(2):185-9. bimortalidad del $17 \%$, infarto de miocardio e insuficiencia renal, así como disfunción multiorgánica y complicaciones mayores en mayor del $10 \%{ }^{14,15}$. El tratamiento percutáneo se ha incrementado en el último tiempo en forma significativa ${ }^{16,17}$. La técnica combinada con coils y stents diversores de flujo es una técnica que combina el tratamiento de los AAR para preservar ramas principales ${ }^{18,20}$. Estos stent de múltiples capas que generan flujos diferentes en el aneurisma y en las ramas no comprometidas.

\section{CONCLUSIÓN}

Los aneurismas renales, aunque infrecuentes son potencialmente mortales, generalmente son asintomáticos y su indicación de exclusión es de $\geq$ a $2 \mathrm{~cm}$. El tratamiento percutáneo utilizando diversas técnicas puede ser efectivos en resolverlos.En aneurismas múltiples, aunque estos involucren ramas principales, la técnica combinada de coils y diversor de flujo, como la aquí presentada, puede excluirlos en forma completa con preservación de sus ramas.
12. 12GonzálezJ, Esteban M, Andrés G, Linares E, Martínez-Salamanca Jl. Renal artery 175 aneurysms. Current urology reports. 2014;15(1):376.

13. 13 Henriksson C, Bjorkerud S, Nilson AE, et al. Natural history of renal artery aneurysm elucidated by repeated angiography and pathoanatomical studies. Eur Urol. 1985. 11(4):244-8.

14. 14 Pfeiffer $T$, Reiher $L$, Grabitz $K$ et al. Reconstruction for renal artery aneurysm: operative techniques and long-term results.J Vasc Surg. 2003 Feb;37(2):293-300.

15. 15 Klausner JQ, Lawrence PF, Harlander-Locke MP, et al. The contemporary management ofrenal artery aneurysms.JVasc Surg. 2015 Apr;61 (4):978-84.

16. 16 Tsilimparis N, Reeves JG, Dayama A, et al. Endovascular vs open repair of renal artery aneurysms: outcomes of repair and long-term renal function.J Am Coll Surg. 2013 Aug;217(2):263-9.

17. 17 Antoniou G, Antoniou S. Endovascular stent graft repair of renal artery aneurysms.Int Angiol. 2011 Oct;30(5):481-7.

18. 18Sfyroeras GS, Dalainas I, Giannakopoulos TG, et al. Flow-divertingstents for the treatment of arterial aneurysms. Journal of vascular surgery.

19. 19 Meyer C, Verrel F, Weyer G, Wilhelm K. Endovascular management of complex renal artery aneurysms using the multilayer stent. Cardiovascular and interventional radiology. 2011; 34(3): 637-41.

20. 20 Mieres J, Cárdenas R, FernándezC, et al. Multiple renal aneurysms involving the main branch. Percutaneous resolution with combined technique of coils and Flow-diverting stent. 2018. CIRSE, September, Copenhagen, Denmark. 